\title{
Risperidone-induced type 2 diabetes presenting with diabetic ketoacidosis
}

\author{
Clarissa Ern Hui Fang', Mohammed Faraz Rafey,2, Aine Cunningham', Sean F Dinneen1,2 \\ and Francis M Finucane ${ }^{1,2}$
}

1Bariatric Medicine Service, Centre for Diabetes, Endocrinology and Metabolism, Galway University Hospitals, Galway Ireland and 2HRB Clinical Research Facility, National University of Ireland Galway, Galway, Ireland
Correspondence should be addressed

to F M Finucane Email

francis.finucane@hse.ie

\section{Summary}

A 28-year-old male presented with 2 days of vomiting and abdominal pain, preceded by 2 weeks of thirst, polyuria and polydipsia. He had recently started risperidone for obsessive-compulsive disorder. He reported a high dietary sugar intake and had a strong family history of type 2 diabetes mellitus (T2DM). On admission, he was tachycardic, tachypnoeic and drowsy with a Glasgow Coma Scale (GCS) of 10/15. We noted axillary acanthosis nigricans and obesity (BMI 33.2 kg/m²). Dipstick urinalysis showed ketonuria and glycosuria. Blood results were consistent with diabetic ketoacidosis (DKA), with hyperosmolar state. We initiated our DKA protocol, with intravenous insulin, fluids and potassium, and we discontinued risperidone. His obesity, family history of T2DM, acanthosis nigricans and hyperosmolar state prompted consideration of T2DM presenting with 'ketosis-prone diabetes' (KPD) rather than T1DM. Antibody markers of beta-cell autoimmunity were subsequently negative. Four weeks later, he had modified his diet and lost weight, and his metabolic parameters had normalised. We reduced his total daily insulin dose from 35 to 18 units and introduced metformin. We stopped insulin completely by week 7. At 6 months, his glucometer readings and glycated haemoglobin (HbA1c) level had normalised.

\section{Learning points:}

- Risperidone-induced diabetic ketoacidosis (DKA) is not synonymous with type 1 diabetes, even in young white patients and may be a manifestation of 'ketosis-prone' type 2 diabetes (KPD).

- KPD is often only confirmed after the initial presentation, when islet autoimmunity and cautious phasing out of insulin therapy have been assessed, and emergency DKA management remains the same.

- As in other cases of KPD, a family history of T2DM and presence of cutaneous markers of insulin resistance were important clinical features suggestive of an alternative aetiology for DKA.

\section{Background}

Patients taking antipsychotic medications have a wellestablished increased risk of developing obesity and type 2 diabetes mellitus (T2DM) (1). A large Danish cohort study found that patients taking second-generation antipsychotics had a $32 \%$ increased risk of developing diabetes, with the risk being 24\% higher in those on risperidone (2). Diabetic ketoacidosis (DKA) is also more common in patients taking antipsychotic drugs (3). This tends to occur within 6 months of initiation of treatment and is more likely with combination antipsychotic therapy $(3,4)$.

Presentation with DKA as a manifestation of type 2 rather than type 1 diabetes is well described (5) and is emphasised in clinical guidelines (6). This 'ketosis-prone diabetes' (KPD) phenotype is characterised by a similar 
initial presentation with DKA, but with transient, nonimmune-mediated beta-cell failure that is often reversible, such that affected patients may be gradually weaned off insulin completely within weeks of presentation (7).

However, awareness of KPD within non-endocrine specialties is variable, with misconceptions that ketoacidosis is synonymous with absolute rather than relative insulin deficiency and with type 1 diabetes. In particular, to our knowledge, risperidone-induced KPD has not previously been described. Moreover, the contribution of the insulin-resistant KPD 'phenotype' to antipsychotic-induced DKA is not well recognised, even in recent studies $(3,4)$.

\section{Case presentation}

A 28-year-old white male presented to our emergency department with a 2-day history of recurrent vomiting and epigastric pain. He had osmotic symptoms with thirst, polyuria and polydipsia for the preceding 2 weeks. His only medical history was of severe depression and obsessivecompulsive disorder, treated with sertraline $350 \mathrm{mg}$ daily, quetiapine $25 \mathrm{mg}$ twice daily, pregabalin $75 \mathrm{mg}$ twice daily and zolpidem $10 \mathrm{mg}$ nocte. Two weeks earlier, he started risperidone $0.5 \mathrm{mg}$ twice daily. He never smoked or drank alcohol. His diet was high in refined carbohydrates. His mother had T2DM, as did a paternal uncle.

On admission, he was drowsy with a GCS of 10/15 (eye opening 3, verbal response 2 and motor response 5 ). Blood pressure was 130/80 $\mathrm{mmHg}$, heart rate 113 beats per minute, respiratory rate 20 breaths per minute, oxygen saturations were $100 \%$ on room air, and he was afebrile. There was no focal neurological deficit and respiratory, cardiovascular and abdominal examinations were otherwise normal. He was obese with a BMI of $33.2 \mathrm{~kg} / \mathrm{m}^{2}$. He had subtle axillary acanthosis nigricans.

\section{Investigation}

At presentation, the patient had a markedly elevated blood glucose and beta-hydroxybutyrate, with very low bicarbonate, as shown in Table 1 . His arterial $\mathrm{pH}$ was 6.89 and $\mathrm{pCO}_{2}$ was 2.9, consistent with severe DKA, with respiratory compensation. He also had a hyperosmolar state, as shown. Moreover, he had an acute kidney injury with elevated urea and creatinine and an estimated glomerular filtration rate of $26 \mathrm{~mL} / \mathrm{min}$ $1.73 / \mathrm{m}^{2}$. Because of his altered level of consciousness, we performed CT and MRI brain and then lumbar puncture, all of which were normal. We noted an elevated alanine aminotransferase (ALT) level, most likely due to nonalcoholic steatohepatosis. His markedly elevated HbA1c at presentation suggested undiagnosed, asymptomatic diabetes that pre-dated his risperidone therapy.

\section{Treatment}

We initiated our DKA protocol, with intravenous insulin, fluids and potassium. All of his psychiatric medications were held because of his low GCS and hyponatraemia on admission, which progressed quickly to hypernatraemia ( $\mathrm{Na} 161 \mathrm{mmol} / \mathrm{L}$ ) with restoration of euglycaemia over $36 \mathrm{~h}$. A nasogastric tube was inserted and feeding commenced, and a urinary catheter was inserted. Empiric antibiotic and antiviral therapy was administered until an acute CNS infection was excluded with imaging and lumbar puncture. On day 3 , he was transitioned from intravenous to subcutaneous insulin glargine (Lantus) 20 units nocte and insulin aspart (NovoRapid) 5 units three times daily with meals. He regained consciousness on day 4 with a GCS of $14 / 15$ (eye movement 4 , verbal response 4 and motor response 6). An EEG was normal. By day 7, his neurological status

Table 1 Anthropometric and metabolic characteristics of patient with risperidone-induced KPD.

\begin{tabular}{|c|c|c|c|}
\hline & At presentation & Day 9 (discharge) & 6 months post admission \\
\hline Weight (kg) & 110 & 89.4 & 76.2 \\
\hline $\mathrm{BMI}\left(\mathrm{kg} / \mathrm{m}^{2}\right)$ & 33.2 & 26.9 & 23 \\
\hline Blood glucose (mmol/L) & 41 & 10 & 5 \\
\hline $\mathrm{HCO}^{-}(\mathrm{mmol} / \mathrm{L})$ & 4.1 & 21.6 & N/A \\
\hline Plasma Na (mmol/L) & 127 & 141 & 145 \\
\hline Plasma Na (mmol/L) (corrected) & 137 & 141 & N/A \\
\hline Plasma osmolality (mosmol/L) & 402 & 294 & 298 \\
\hline Beta-hydroxybutyrate ( $\mu \mathrm{mol} / \mathrm{L})$ & 4450 & 0.1 & 0.2 \\
\hline Urea (mmol/L) & 13.9 & 2.3 & 2.8 \\
\hline Creatinine (mmol/L) & 273 & 67 & 88 \\
\hline $\operatorname{ALT}(\mu / L)$ & 96 & 25 & 31 \\
\hline $\mathrm{HbA1c}(\mathrm{mmol} / \mathrm{mol})$ & 94 & N/A & 35 \\
\hline
\end{tabular}


had normalised and the psychiatry team reintroduced sertraline $300 \mathrm{mg}$ od and started promethazine $15 \mathrm{mg}$ nocte. He was discharged on day 9 with regular telephone contact with the diabetes nurse specialist for subsequent insulin dose adjustment.

\section{Outcome and follow-up}

When the patient was followed up 4 weeks later in clinic, he reported modifying his diet substantially, cutting out sugary foods. His weight had come down to $89.4 \mathrm{~kg}$ (BMI $26.9 \mathrm{~kg} / \mathrm{m}^{2}$ ), a reduction of $20.6 \mathrm{~kg}$, while his glucometer readings had normalised. We reduced his total daily insulin dose from 35 to 18 units and added metformin $500 \mathrm{mg}$ twice daily, increasing to $1000 \mathrm{mg} 2$ weeks later and gradually titrating his insulin dose to zero, 7 weeks after his initial presentation. At 6 months, his BMI, glucometer readings, HbA1c and ALT had normalised (Table 1). Metformin was stopped completely. His antiislet cell and anti-glutamic acid decarboxylase antibodies were subsequently negative.

\section{Discussion}

Based on the transient requirement for insulin, absence of islet cell autoimmunity, presence of hyperosmolarity, excess adiposity, strong family history, probable hepatic steatosis, acanthosis and excellent response to metformin and lifestyle modification, we diagnosed risperidoneinduced KPD. (We thought that risperidone was more likely than quetiapine to be the offending agent because of the temporal association between its initiation and symptom onset and its worse impact on glucose homeostasis (2)). This highlights that risperidone-induced DKA is not synonymous with T1DM and permanent, autoimmune destruction of beta cells, but may arise from reversible beta-cell failure in patients with features of T2DM. It is likely that our patient had asymptomatic and undiagnosed diabetes for at least several weeks prior to starting risperidone given his $\mathrm{HbA} 1 \mathrm{c}$ of $94 \mathrm{mmol} /$ mol, and that the drug somehow aggravated a further deterioration in glycaemic control, manifesting with osmotic symptoms and DKA.

The pathophysiology of absolute, albeit transient beta-cell failure in KPD may involve glucotoxicity secondary to subacute or chronic hyperglycaemia, probably aggravated by underlying insulin resistance (8). It is likely that many antipsychotic-treated patients who have presented with DKA and subsequently stopped insulin therapy with restoration of normoglycaemia had
KPD. Our approach (as with any patient with DKA) was to initiate treatment with insulin acutely and to consider introducing an insulin sensitiser (metformin) and reducing the insulin dose only after glycaemic control was restored and severe metabolic derangements had fully resolved.

We did not rely on islet autoantibodies in making a provisional diagnosis of KPD, as these results are usually only available weeks after the initial presentation. Nonetheless, their utility is emphasised in several proposed classification schemes for KPD, in order to predict which patients may be weaned off insulin (9). Others suggest that BMI is helpful (8), but we regard it as a crude measure of excess adiposity. The ' $\alpha \beta$ ' system (9), which considers beta-cell function (measured with C-peptide) as well as islet autoimmunity, seems like a rational approach, though we do not routinely measure C-peptide in all potential KPD patients and in the acute phase of beta-cell failure, this may be low. While clinical guidelines have started to emphasise KPD as an important consideration in patients presenting with DKA, consensus on the optimal way to diagnose and manage KPD is lacking. Our practice is to be guided by clinical features apparent at the outset. Although not emphasised in guidelines, we have found that a family history of T2DM and cutaneous markers of insulin resistance are strongly suggestive of KPD (10).

\section{Declaration of interest}

Francis Finucane has received honoraria, travel grants and has served on advisory boards for Novo Nordisk, Eli Lilly, Pfizer Inc., Sanofi-Aventis, Astra Zeneca, Merck-Serono, Boehringer Ingelheim, Janssen and Novartis. Clarissa Fang, Aine Cunningham, Sean Dinneen and Mohammed Rafey have no conflicts of interest to declare.

\section{Funding}

This research did not receive any specific grant from any funding agency in the public, commercial or not-for-profit sector.

\section{Patient consent}

Written informed consent for publication of their anonymised clinical details was obtained from this patient.

\section{Author contribution statement}

Clarissa Ern Hui Fang helped in description of case, collation of data, drafting manuscript. Mohammed Faraz Rafey helped in management of case, collection of data, reviewing manuscript. Aine Cunningham helped in management of case, reviewing manuscript. Sean F Dinneen helped in management of case, reviewing of manuscript. Francis M Finucane helped in collation of data, drafting of manuscript, supervision of project. 


\section{References}

1 American Diabetes Association,, American Psychiatric Association,, American Association of Clinical Endocrinologists, \& North American Association for the Study of Obesity. Consensus development conference on antipsychotic drugs and obesity and diabetes. Diabetes Care 200427 596-601. (https://doi.org/10.2337/ diacare.27.2.596)

2 Kessing LV, Thomsen AF, Mogensen UB \& Andersen PK. Treatment with antipsychotics and the risk of diabetes in clinical practice. British Journal of Psychiatry 2010197 266-271. (https://doi. org/10.1192/bjp.bp.109.076935)

3 Polcwiartek C, Vang T, Bruhn CH, Hashemi N, Rosenzweig M \& Nielsen J. Diabetic ketoacidosis in patients exposed to antipsychotics: a systematic literature review and analysis of Danish adverse drug event reports. Psychopharmacology 2016233 3663-3672. (https://doi. org/10.1007/s00213-016-4411-x)

4 Vuk A, Kuzman MR, Baretic M \& Osvatic MM. Diabetic ketoacidosis associated with antipsychotic drugs: case reports and a review of literature. Psychiatria Danubina 201729 121-135. (https://doi. org/10.24869/psyd.2017.121)

5 Banerji MA, Chaiken RL, Huey H, Tuomi T, Norin AJ, Mackay IR, Rowley MJ, Zimmet PZ \& Lebovitz HE. GAD antibody negative
NIDDM in adult black subjects with diabetic ketoacidosis and increased frequency of human leukocyte antigen DR3 and DR4. Flatbush diabetes. Diabetes 199443 741-745. (https://doi. org/10.2337/diab.43.6.741)

6 American Diabetes Association. Diabetes care in the hospital: standards of medical care in diabetes-2018. Diabetes Care 2018 41:S144-S151. (https://doi.org/10.2337/dc18-S014)

7 Balasubramanyam A, Nalini R, Hampe CS \& Maldonado M. Syndromes of ketosis-prone diabetes mellitus. Endocrine Reviews 2008 29 292-302. (https://doi.org/10.1210/er.2007-0026)

8 Umpierrez GE, Woo W, Hagopian WA, Isaacs SD, Palmer JP, Gaur LK, Nepom GT, Clark WS, Mixon PS \& Kitabchi AE. Immunogenetic analysis suggests different pathogenesis for obese and lean AfricanAmericans with diabetic ketoacidosis. Diabetes Care 199922 1517-1523. (https://doi.org/10.2337/diacare.22.9.1517)

9 Balasubramanyam A, Garza G, Rodriguez L, Hampe CS, Gaur L, Lernmark A \& Maldonado MR. Accuracy and predictive value of classification schemes for ketosis-prone diabetes. Diabetes Care 2006 29 2575-2579. (https://doi.org/10.2337/dc06-0749)

10 Small C, Egan AM, Elhadi EM, O'Reilly MW, Cunningham A \& Finucane FM. Diabetic ketoacidosis: a challenging diabetes phenotype. Endocrinology, Diabetes and Metabolism Case Reports 2017 Article ID: EDM-16-0109. (https://doi.org/10.1530/EDM-16-0109) 